\title{
A simple method for electrical machine's mechanical parameter extraction
}

\author{
Farzin Asadi ${ }^{1}$ *, Nurettin Abut ${ }^{2}$, Ismet Kandilli ${ }^{3}$ \\ ${ }_{1}^{1}$ Department of Mechatronics, Engineering Faculty, Kocaeli University, Kocaeli, Turkey \\ 2Department of Electrical Engineering, Engineering Faculty, Kocaeli University, Kocaeli, Turkey \\ ${ }^{3}$ Department of Electronics and Automation, Kocaeli University, Kocaeli, Turkey
}

\section{ARTICLE IN F O}

\section{Article history:}

Received 2 December 2016

Received in revised form

27 February 2017

Accepted 28 February 2017

\section{Keywords:}

Coefficient of viscous friction

Motion control system

Motor's mechanical parameters

Rotor's moment of inertia

\begin{abstract}
A B S T R A C T
Electrical motors are one of the most important key components of industry. While motors can be divided in so many groups, mathematical description of all of them is divided into two subsystems: Electrical and mechanical subsystem. Mechanical subsystem is usually composed of rotor inertia, external load and friction in bearing of rotor. Friction is usually modeled as viscous friction, i.e. linearly dependent on angular speed. Rotor inertia J) and coefficient of viscous friction (B) are needed in order to model mechanical subsystem of motor. When motor is used in high performance close loop motion control systems, an accurate model of motor is required for system analysis and design. This paper suggests a novel method for measuring rotor's J and B for such applications. There is no restriction on the type of motor under test. Studied method, needs no sensor so no friction is added to motor. Only a digital camera is required. There is no need to open the motor case and remove motor's rotor in this method. Proposed method has been tested in laboratory and practical results shows effectiveness of suggested method.
\end{abstract}

(C) 2017 The Authors. Published by IASE. This is an open access article under the CC BY-NC-ND license (http://creativecommons.org/licenses/by-nc-nd/4.0/).

\section{Introduction}

Electrical energy comes from renewable and non-renewable sources. Some of the electrical energy's advantages over other forms of energy are as follows:

- Relatively cheap and available almost everywhere.

- Easiness of transport from one point to the other. Only a pair of wire is needed. Also, transfer of large amount of energy can be done with high efficiency.

- When converted to mechanical power, no smoke or other forms of air pollution is produced unlike internal combustion engines. So, electrical motors can work in closed environment such as homes and offices.

- Electrical energy can be converted to almost all other forms of energy easily.

- In addition to the aforementioned benefits, electrical energy has some drawbacks:

- Storing large amounts of electrical energy is not an easy task.

\footnotetext{
* Corresponding Author.

Email Address: farzin.asadi@kocaeli.edu.tr (F. Asadi) https://doi.org/10.21833/ijaas.2017.04.024

2313-626X/C 2017 The Authors. Published by IASE.

This is an open access article under the CC BY-NC-ND license

(http://creativecommons.org/licenses/by-nc-nd/4.0/D
}

- When used improperly can lead to death.

Transformation of electrical power to mechanical power can be done with the aid of electrical motors. Fig. 1 shows different categories of electrical motors.

Although different names can be seen in Fig. 1, it must be emphasized that working principles of all these families are the same: A conductor of length $l$, carrying current I, experience a force $\mathrm{F}$ in presence of magnetic field $\mathrm{B}$ which can be calculated as: $F=$ I. $(l \times B)$.

Generally speaking, motor's generated torque must overcome two resistive counter torques: load's torque and friction. Equation of motion can be written as (Eq. 1):

$\tau-\tau_{\text {load }}-B \omega=J \frac{d}{d t}(\omega)$

where,

$\tau$ : Motor's generated torque,

$\tau_{\text {load }}$ : Load's torque

$B$ : Coefficient of viscous friction,

$\omega$ : Load's angular speed,

J: Moment of inertia.

Developing a method for motor's mechanical parameter extraction (B and J in Eq. 1) is the aim of this paper. 


\subsection{Literature review}

There are several analytical and experimental methods to find moment of inertia in literature
(Boldea and Nasar, 1986; Vas, 2001; Cornea et al., 2007; Zverlov et al., 1999). Traditional methods are:

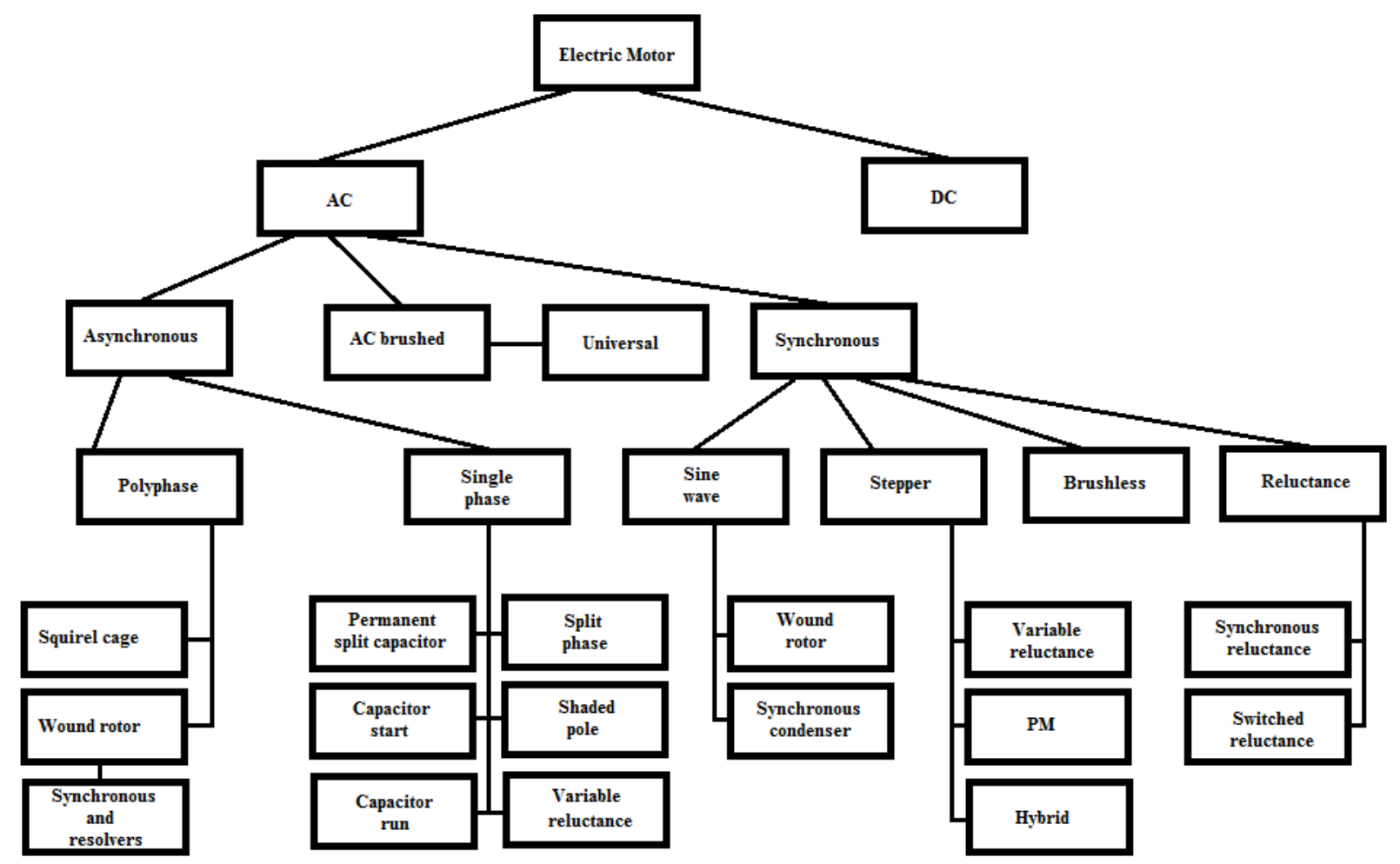

Fig. 1: Different categories of electrical motors

- Torsion oscillation test,

- Pendulum test,

- Retardation test.

The torsion oscillation test needs the removal of rotor. Rotor is hanging from a torsion wire and is rotated around rotor axes. Period of oscillation, determines the inertia. In practice, instead of aforementioned procedure, inertia of Motor under Test (MUT) is calculated using a comparison with a known rotor (Eq. 2) (Ilina, 2011):

$J_{\text {rotor }}=J_{\text {known }} \times\left(\frac{T_{\text {rotor }}}{T_{\text {known }}}\right)^{2}$

Pendulum test and retardation test do not need rotor removal. In pendulum test, a bar of length $l$ is connected to the rotor's shaft. Mass of $m$ is connected to free end of bar. Bar can rotate around motor's shaft. Using period of oscillations (T), inertia can be calculated as (Eq. 3) (Ilina, 2011):

$J_{\text {rotor }}=m \cdot l \cdot\left(\frac{T^{2} \cdot g}{4 \cdot \pi^{2}}-l\right)$

Retardation test is based on rotating the shaft up to a speed over the nominal speed. Machine is let free to slow down and speed is recorded with a data acquisition system.

After some algebra, inertia can be calculated using Eq. 4 (Ilina, 2011):
$J_{\text {rotor }}=-\frac{P_{m n}}{\left(\frac{\pi}{30}\right)^{2} \times n_{n} \times \frac{d}{d t}(n)_{n=n_{n}}}$

$\mathrm{M}_{\mathrm{mn}}$ is the torque corresponding to rated mechanical power losses, $\mathrm{n}_{\mathrm{n}}$ is rated speed, $\frac{d}{d t}\left(n_{n}\right)$ represents the ramp of the line tangent $A D$ in point $A$ to the curve $n(t)$ and $P_{m n}$ is the mechanical losses at rated speed. Fig. 2 shows the typical retardation test result for this method.

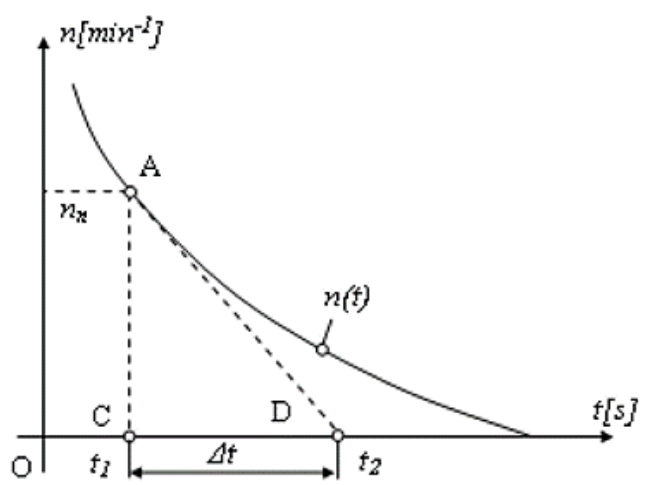

Fig. 2: Typical deceleration curve for retardation test

Also, more advanced techniques of inertia identification using observer (Awaya et al., 1992; Lee et al., 2004; Yang and Deng, 2005), extended Kalman filtering (Kwon et al., 2006; Boileau et al., 2008; Hong et al., 1996), model reference adaptive method (Andoh, 2008; Okamura et al., 1995), gradient descent method (Boileau et al., 2011) are available in 
literature. These methods, generally needs a more complicated hardware.

\section{Suggested method}

In order to measure rotor moment of inertia (J) and coefficient of viscous friction (B) following two step procedures is suggested:

\subsection{Measurement of $\frac{J}{B}$ Ratio}

In this test, unloaded MUT is turned on and off is turned on and after reaching the nominal speed is turned off.

A pointer is attached to the motor's shaft. Shaft's deceleration is recorded with the aid of a digital camera. Obtained video is processed with video editing software so the deceleration interval's length (Interval between de energizing the motor and full stop of pointer attached to shaft) can be measured $\left(\Delta T_{1}\right)$.

When motor is de energized, equation of motion can be written as (Eqs. 5 and 6):

$J \frac{d}{d t}(\omega)+B \omega=0$

solution of Eq. 5 is:

$\omega=\omega_{0} \times e^{-\frac{B}{J} t}$

Eq. 6 is approximately zero for $t \geq \frac{5 \times J}{B}$. So, $\Delta T_{1} \approx$ $\frac{5 \times J}{B}$ or $\frac{J}{B} \approx \frac{\Delta T_{1}}{5}$. So, ratio of $\frac{J}{B}$ can be approximated with the aid of this test.

\subsection{Constant torque test}

In this test, motor is disconnected from electrical source of energy and then a constant specific weight $\mathrm{W}$ is hanging from motor shaft with a short thread. Aim of this test is to apply a constant torque ( $\left.\tau_{\text {thread }}\right)$ to motor's shaft and study its motion under this condition.

Torque applied to motor's shaft can be calculated as Eq. 7:

$\tau_{\text {thread }}=\frac{D}{2} \times W$

Where D is motor's shaft diameter. If $\mathrm{W}$ is large enough rotor starts rotation. Rotor rotation is recorded with the aid of a digital camera. Using slow motion capability of video processing software, shaft's motion can be studied carefully. Pointer which is attached to motor's shaft and a dial make it possible to see shaft's position in each instant of time. Obtained video, is analyzed with video editing software in order to extract rotor's position as a function of time. Equation of motion in presence of a constant torque $\tau_{\text {thread }}$ is (Eqs. 8-10):

$\tau_{\text {thread }}-B \omega=J \frac{d}{d t} \omega$ $\omega=\frac{\tau_{\text {thread }}}{B}\left(1-e^{\frac{B}{J} t}\right)$
$\omega=\frac{\tau_{\text {thread }}}{B}\left(1-e^{\frac{5}{\Delta T_{1}} t}\right)$

If $\frac{B}{J} t \ll 1$ equivalently $t \ll \frac{B}{J} \quad$ Eq. 10 can be approximated as (Eq. 11):

$\omega \approx \frac{\tau_{\text {thread }}}{J} \times t$

\section{Extraction of parameters}

After doing the above tests, mechanical parameters (B and J) can be extracted. As mentioned, $\frac{J}{B} \approx \frac{\Delta T 1}{5}$ or equivalently (Eq. 12 ):

$J \approx \frac{\Delta T 1}{5} \times B$

If $B$ is known, J can be calculated using this equation. In order to calculate B, Eq. 10 and data obtained from constant torque test is used. So problem of finding $\mathrm{B}$ is converted to a curve fitting problem which can be solved using Least Square Method (LSM). After determining value of B using LSM, rotor's inertia can be calculated using Eq. 9.

\section{Setup}

In order to test aforementioned procedure, a digital camera, is used to record rotors motions. Dial is pasted on the motor's body. A water bottle connected with a thread to motor's shaft, is used as load (Fig. 3). Volume of the water in bottle is set such that rotor can start rotation. Motor can be de energized using a stop button. When motor's $\frac{J}{B}$ ratio is measured, camera records the moment which user press stop button so measurement of deceleration interval can be done with respect to the moment which energy is disconnected.

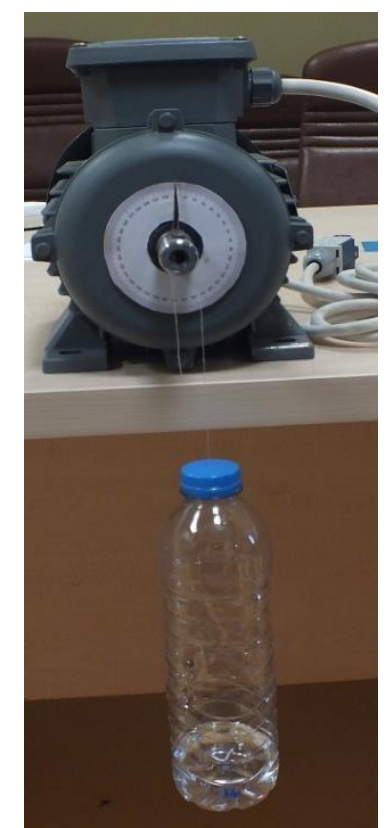

Fig. 3: Setup for constant torque test. 


\section{Practical results}

Applying the aforementioned procedure to a 380 volt, $0.75 \mathrm{KW}, 3$-phase motor with $\mathrm{D}=18.5 \mathrm{~mm}$ lead to $\Delta T_{1}=6.74 \mathrm{sec}$. Constant torque test is done with $\mathrm{W}=281.19 \mathrm{gr}$ which lead to $\tau_{\text {thread }}=0.0255 \mathrm{~N} . \mathrm{m}$. Table 1 shows rotor's positions obtained for constant torque test. Also, Fig. 4, shows the plotted data.

Table 1: Rotor position vs. time for constant torque of

\begin{tabular}{cccc}
\hline No. & Time(sec.) & $\begin{array}{c}\text { Shaft's angle (in } \\
\text { Degrees) }\end{array}$ & $\begin{array}{c}\text { Shaft's angle } \\
\text { (in Radians) }\end{array}$ \\
\hline 1 & 0.000 & 0 & 0 \\
2 & 0.135 & 0 & 0 \\
3 & 0.383 & 5 & 0.087 \\
4 & 0.570 & 9 & 0.157 \\
5 & 0.645 & 14 & 0.244 \\
6 & 0.780 & 21 & 0.367 \\
7 & 0.858 & 24 & 0.419 \\
8 & 0.925 & 29 & 0.506 \\
9 & 1.053 & 38 & 0.663 \\
10 & 1.110 & 43 & 0.751 \\
11 & 1.248 & 55 & 0.960 \\
12 & 1.323 & 60 & 1.047 \\
13 & 1.390 & 65 & 1.135 \\
14 & 1.458 & 75 & 1.309 \\
15 & 1.518 & 80 & 1.396 \\
\hline
\end{tabular}

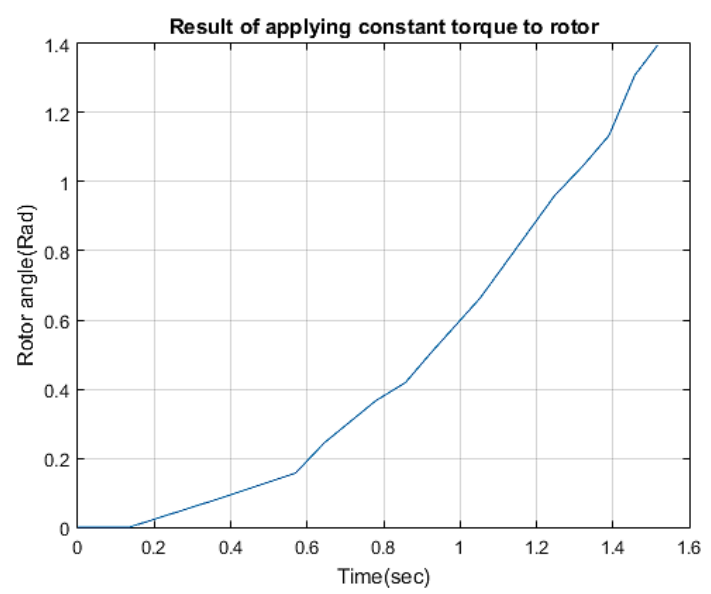

Fig. 4: Position of rotor in constant torque test

Fitting a second order polynomial to this data using Matlab ${ }^{\circledR}$ gives the following results (Eq. 13):

$\theta=0.6297 \times t^{2}-0.03138 \times t \quad-0.003571$

where rotor's angle $(\theta)$ is in radians and $t$ is in seconds. Fig. 5 shows fitted curve and data points on the same graph. Result of curve fitting is shown in Fig. 6. 14):

Using Eq. 13 angular speed can be found as (Eq.

$\omega=\frac{d \theta}{d t}=1.2594 \times t-0.03138$

Where $\omega=\frac{d \theta}{d t}$ is in $\frac{R a d}{s}$ and $t$ is in seconds. We want to find the best coefficient of viscous friction (B) such that difference between Eqs. 10 and 14 is minimum. So, problem can be converted to an optimization problem:
Find B such that (Eqs. 10 and 14) is minimized.

Application of LSM to the above optimization problem leads to (Eq. 15):

$B=0.01025$ N.m.s.

and J can be calculated as (Eq. 16):

$\mathrm{J}=0.0138 \mathrm{~kg} \cdot \mathrm{m}^{2}$.

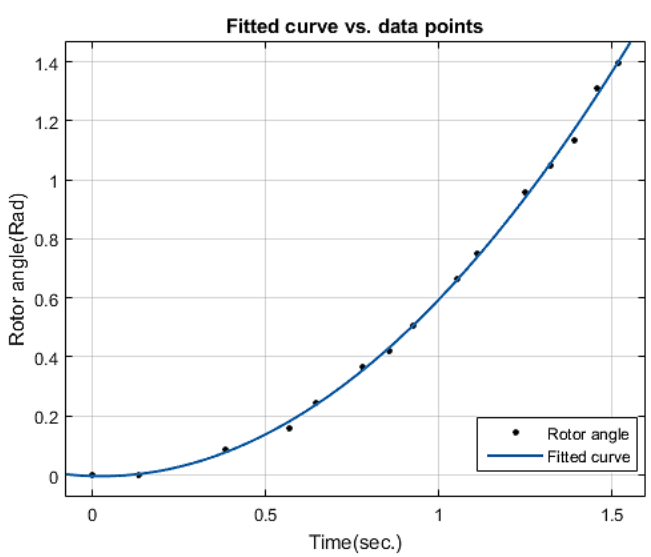

Fig. 5: Fitted curve vs. data points

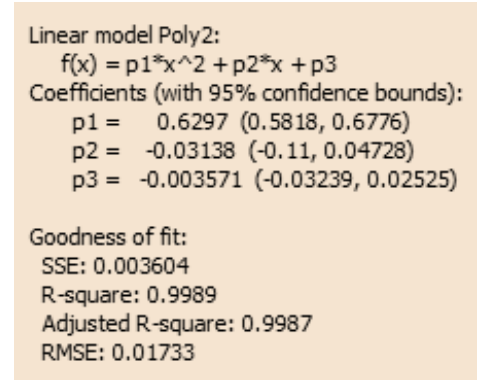

Fig. 6: Results of curve fitting

Fig. 7, shows rotor's angular speed predicted by Eq. 14 vs. values predicted by best fit (Eq. 10 with $\mathrm{B}=0.01025$ N.m.s) at time instances of Table 1. Fig. 8, shows statistical properties of best fit.

\subsection{Verification of obtained results}

In order to ensure correctness of the method, motor's case has been opened rotor's inertia has been calculated with the formulas of inertia for cylindrical sections. Fig. 9 shows motor's parts. Rotor's mass measured as $2.582 \mathrm{Kg}$. Rotor's inertia has been calculated as shown in Eq. 17.

$$
\mathrm{B}=0.0015 \mathrm{Kg} \cdot \mathrm{m}^{2}
$$

This shows a tremendous error between what has been predicted by aforementioned method. In order to find the reason, look more carefully to model that has been assumed for rotor's motion which has been written here again for easiness of reference:

$J \frac{d^{2}}{d t^{2}}(\theta)+B \frac{d}{d t}(\theta)=\tau_{\text {thread }} \theta(0)=\dot{\theta}(0)=0$. 
This equation suggests that there is a movement, i.e. change in rotor's angular position for all values of $\tau_{\text {thread }}$. But this is not what seen in real world.

When a torque is applied to motor's shaft there is no movement up to a certain threshold torque $\left(\tau_{\text {threshold }}\right)$.

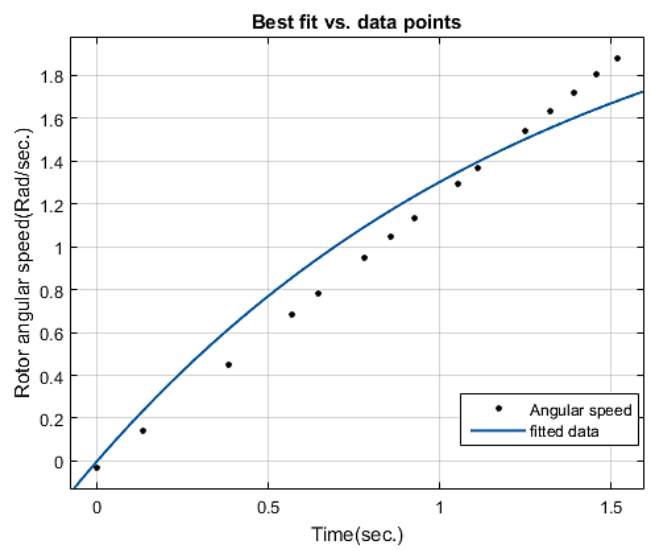

Fig. 7: Eq. 7 with $B=0.01025$ vs. Eq. 14

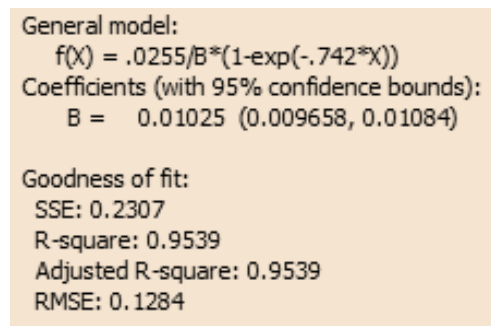

Fig. 8: Statistical properties of best fit

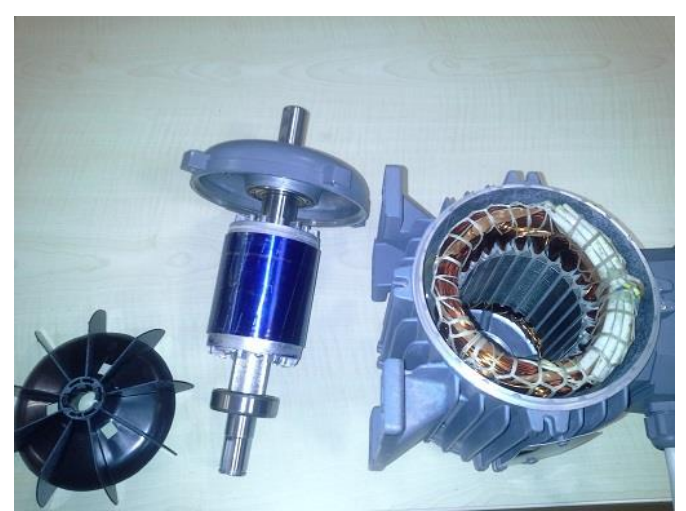

Fig. 9: Opening motor's case

When $w<260 \mathrm{gr}$ (see Eq. 4) there is no rotation in the motor's shaft so when $w=281.19 \mathrm{gr}$ is applied as in the above tests, applied torque to motor's shaft, which cause shaft's acceleration is not $0.28119 \times g \times \frac{D}{2}=0.0255$ but $(0.28119-0.260) \times$ $g \times \frac{D}{2}=0.002 \mathrm{~N} . \mathrm{m} \quad$ where $\quad g=9.81 \frac{\mathrm{m}}{\mathrm{s}^{2}}$ is gravitational field intensity and $D=18.5 \mathrm{~mm}$ is motor's shaft diameter.

So, equation of motion can be corrected as (Eq. 18):

$J \frac{d^{2}}{d t^{2}}(\theta)+B \frac{d}{d t}(\theta)=\tau_{\text {thread }}-\tau_{\text {threshold }} \quad \theta(0)=$ $\dot{\theta}(0)=0$
Here, aforementioned procedure is modified as follows.

\subsection{Modification of method}

First, Table 1 data is fitted on a Fourier model (Eq. 19):

$\theta=7.371-7.372 \times \cos (0.4252 \times t)-0.1434 \times$

$\sin (0.4252 \times t)$

Data points of Table 1 and Eq. 19, are shown on the same graph in Fig. 10, also statistical properties of fit can be seen in Fig. 11.

Using Eq. 18, motion equation can be written in matrix form as (Eq. 20):

$\left[\frac{d}{d t^{2}}(\theta) \frac{d}{d t}(\theta)\right] \times\left[\begin{array}{l}J \\ B\end{array}\right]=\left[\tau_{\text {thread }}-\tau_{\text {threshold }}\right]$

Matrix $\mathrm{R}=\left[\frac{d}{d t^{2}}(\theta) \quad \frac{d}{d t}(\theta)\right]$ is repressor matrix and $P=\left[\begin{array}{l}J \\ B\end{array}\right]$ parameter matrix. According to Table 1 , 15 , there are data points $t_{1}, t_{2}, t_{3}, \ldots, t_{15}$. If $R$ is calculated for $t_{1}, t_{2}, t_{3}, \ldots, t_{15}$ with $\theta$ given by Eq. 20 , a $15 \times 2$ matrix is obtained (Eq. 21):

$\mathrm{R}=\left[\begin{array}{cc}1.3328 & -0.0610 \\ 1.3321 & 0.1190 \\ 1.3194 & 0.4474 \\ 1.3001 & 0.6931 \\ 1.2900 & 0.7902 \\ 1.2686 & 0.9630 \\ 1.2545 & 1.0608 \\ 1.2410 & 1.1450 \\ 1.2128 & 1.3015 \\ 1.1989 & 1.3708 \\ 1.1628 & 1.5332 \\ 1.1414 & 1.6196 \\ 1.1212 & 1.6960 \\ 1.1000 & 1.7710 \\ 1.0805 & 1.8364\end{array}\right]$

Also $C=\left[\tau_{\text {thread }}-\tau_{\text {threshold }}\right]$ is a constant $15 \times$ 1 matrix.

$C=\left[\begin{array}{l}0.002 \\ 0.002 \\ 0.002 \\ 0.002 \\ 0.002 \\ 0.002 \\ 0.002 \\ 0.002 \\ 0.002 \\ 0.002 \\ 0.002 \\ 0.002 \\ 0.002 \\ 0.002 \\ 0.002\end{array}\right]$

As seen from Eq. 20, there are only 2 unknowns where there are 15 equations. This system can't be solved using matrix inversion techniques because coefficient matrix is not a square matrix. Instead, Moore - Penrose pseudo inverse can be used (Eq. 23):

$\tilde{R}=\left(R^{t} \times R\right)^{-1} \times R^{t}$ 
where, $\tilde{R}$ and $R^{t}$ shows Moore - Penrose pseudo inverse of $R$ and transpose of matrix $R$, respectively. So, matrix $P$ can be calculated as (Eq. 24):

$P=\left[\begin{array}{l}J \\ B\end{array}\right]=\tilde{R} \times C$

Following results are obtained after doing the calculations (Eq. 25):

$P=\left[\begin{array}{l}J \\ B\end{array}\right]=\left[\begin{array}{l}0.0015 \\ 0.0002\end{array}\right]$

Obtained value for J is the same as Eq. 17.

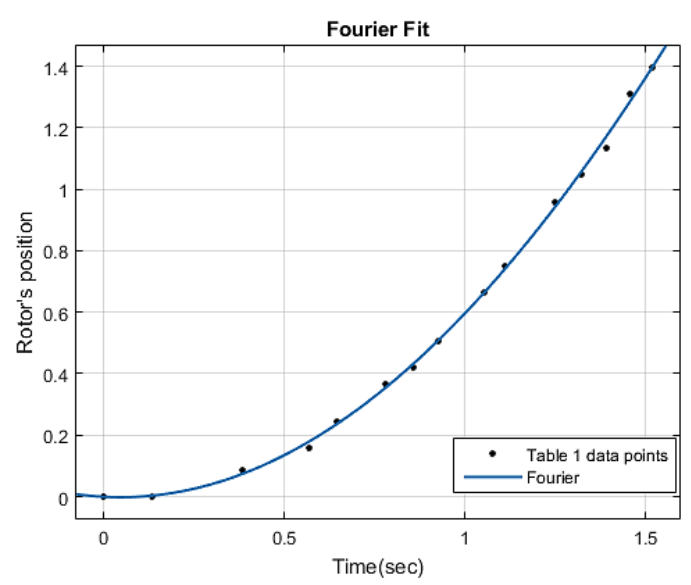

Fig. 10: Fitting Table 1 data to a sinusoidal model

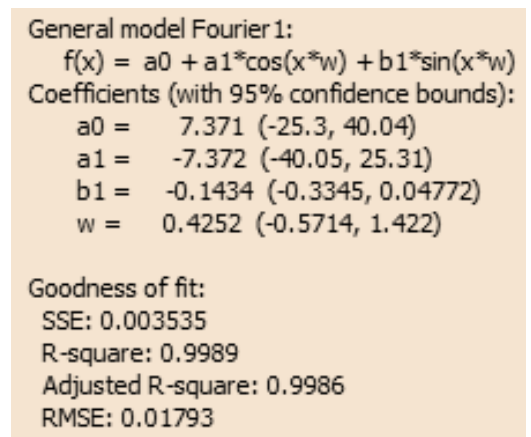

Fig. 11: Statistical properties of Fourier fit

\section{Conclusion}

Motor's electrical and mechanical parameters are important in analyzing and designing High performance motion control systems. In this paper a novel method for motor's mechanical parameter extraction has been studied. There is no need to opening the motor's case and removal of the rotor in this method. No sensor is attached to motor under test so no external friction is added to the system. Proposed method is quite easy and cheap.

\section{References}

Andoh F (2008). Inertia identification method based on the product of the integral of torque reference input and motor speed. In the IEEE International Conference on Control Applications, IEEE, San Antonio, USA: 1151-1158. https://doi.org/10.1109/CCA.2008.4629585

Awaya I, Kato Y, Miyake I, and Ito M (1992). New motion control with inertia identification function using disturbance observer. In the International Conference on Industrial Electronics, Control, Instrumentation, and Automation: Power Electronics and Motion Control, IEEE, San Diego, USA: 77-81. https://doi.org/10.1109/IECON.1992.254601

Boileau T, Leboeuf N, Nahid-Mobarakeh B, and Meibody-Tabar F (2011). Online identification of PMSM parameters: Parameter identifiability and estimator comparative study. IEEE Transactions on Industry Applications, 47(4): 1944-1957.

Boileau T, Nahid-Mobarakeh B, and Meibody-Tabar F (2008). Online identification of PMSM parameters: Model-reference vs EKF. In the IEEE Industry Applications Society Annual Meeting (IAS '08), IEEE, Edmonton, Canada: 1-8. https://doi.org/10.1109/08IAS.2008.176

Boldea I and Nasar S (1986). Electric machine dynamics. MacMillan Publishing, New York, USA.

Cornea O, Sorândaru C, and Musuroi O (2007). Experimental determination of the moment of inertia for switched reluctance motor. In the International Symposium of Electrical Engineering and Energy Converters, ELS, Suceava, Romania.

Hong SJ, Kim HW, and Sul SK (1996). A novel inertia identification method for speed control of electric machine. In the $22^{\text {nd }}$ International Conference on Industrial Electronics, Control, and Instrumentation, IEEE, Taipei, Taiwan, 2: 1234-1239. https://doi.org/10.1109/IECON.1996.566056

Ilina ID (2011). Experimental determination of moment to inertia and mechanical losses vs. speed, in electrical machines. In the $7^{\text {th }}$ International Symposium on Advanced Topics in Electrical Engineering (ATEE), IEEE, Bucharest, Romania: 1-4.

Jiaoyan L, Yuwen H, and Wenqi L (2011). Research on inertia identification performance of permanent magnet servo systems based on gradient algorithm. Acta Aeronautica et Astronautica Sinica, 32(3): 488-496.

Kwon TS, Sul SK, Nakamura H, and Tsuruta K (2006). Identification of the mechanical parameters for servo drive. In the Conference Record of the 2006 on Industry Applications, $41^{\text {st }}$ IAS Annual Meeting, IEEE, Tampa, Florida, USA, 2: $905-$ 910. https://doi.org/10.1109/IAS.2006.256632

Lee KB, Yoo JY, Song JH, and Choy (2004). Improvement of low speed operation of electric machine with an inertia identification using ROELO. In the IEE Proceedings-Electric Power Applications, IET, 151(1): 116-120. https://doi.org/ 10.1049/ip-epa:20031009

Okamura Y, Chun Y, and Hori Y (1995). Inertia Moment Identification in the Average Speed Type Instantaneous Speed Observer. IEEJ Transactions on Industry Applications, 115(2): 130-135.

Vas P (2001). Parameter estimation, condition monitoring, and diagnosis of electrical machines. Oxford University Press, Oxford, UK.

Yang SM and Deng YJ (2005). Observer-based inertial identification for auto-tuning servo motor drives. In the Conference Record of the 2005 on Industry Applications, $40^{\text {th }}$ IAS Annual Meeting, IEEE, Hong Kong, China, 2: 968-972. https://doi.org/10.1109/IAS.2005.1518467

Zverlov BV, Lisov AA, Sokolov BP, and Subbotin AA (1999). Determination of the mechanical losses of electrical rotor machines and the level of degradation. Measurement Techniques, 42(4): 390-393. 